\title{
What do Cells and Photovoltaic Materials Have in Common? TOF-SIMS Tandem MS Imaging and the Identification of Chemistry at $<100 \mathrm{~nm}$ Resolving Power
}

\author{
Gregory L. Fisher ${ }^{1}$ \\ 1. Physical Electronics, Chanhassen, MN 55317, USA
}

The fundamental limitation of rapid, high lateral resolution mass spectrometry (MS) imaging, e.g. TOFSIMS, has been a lack of capacity for confident identification of surface chemistry. A unique TOF-TOF imaging mass spectrometer allows TOF-SIMS ( $\left.\mathrm{MS}^{1}\right)$ imaging and tandem MS $\left(\mathrm{MS}^{2}\right)$ imaging to be achieved simultaneously and in parallel as illustrated by the data shown in Figure 1. Secondary ions for $\mathrm{MS}^{1}$ and $\mathrm{MS}^{2}$ analysis are produced from the same area of the surface by a pulsed and digitally rasterscanned primary ion nanoprobe. Unique and one-of-a-kind samples may be characterized without undesired erosion or degradation; even sub-monolayer 2D films are readily characterized. Monoisotopic precursor ion selection combined with kilo-electron volt (keV) collision-induced dissociation (CID) enables the identification and structural elucidation of surface-bound moieties as exemplified in Figure 2. This relatively new analytical capability has been brought to bear for straightforward molecular identification as well as multifaceted studies involving surface modification, polymers, composites, catalysis, forensic / failure analysis, biology and pharmaceuticals. Practical lateral resolution $(\Delta \boldsymbol{\ell})$ in both $\mathrm{MS}^{1}$ and $\mathrm{MS}^{2}$ images is always $\leq 1 \mu \mathrm{m}$, and can be $<100 \mathrm{~nm}$ when desired. Two subjects of study will be the focus of this presentation: three-dimensional imaging of cellular organelles (see Figure 3 ), and threedimensional characterization of multilayer organic photovoltaic (OPV) materials.

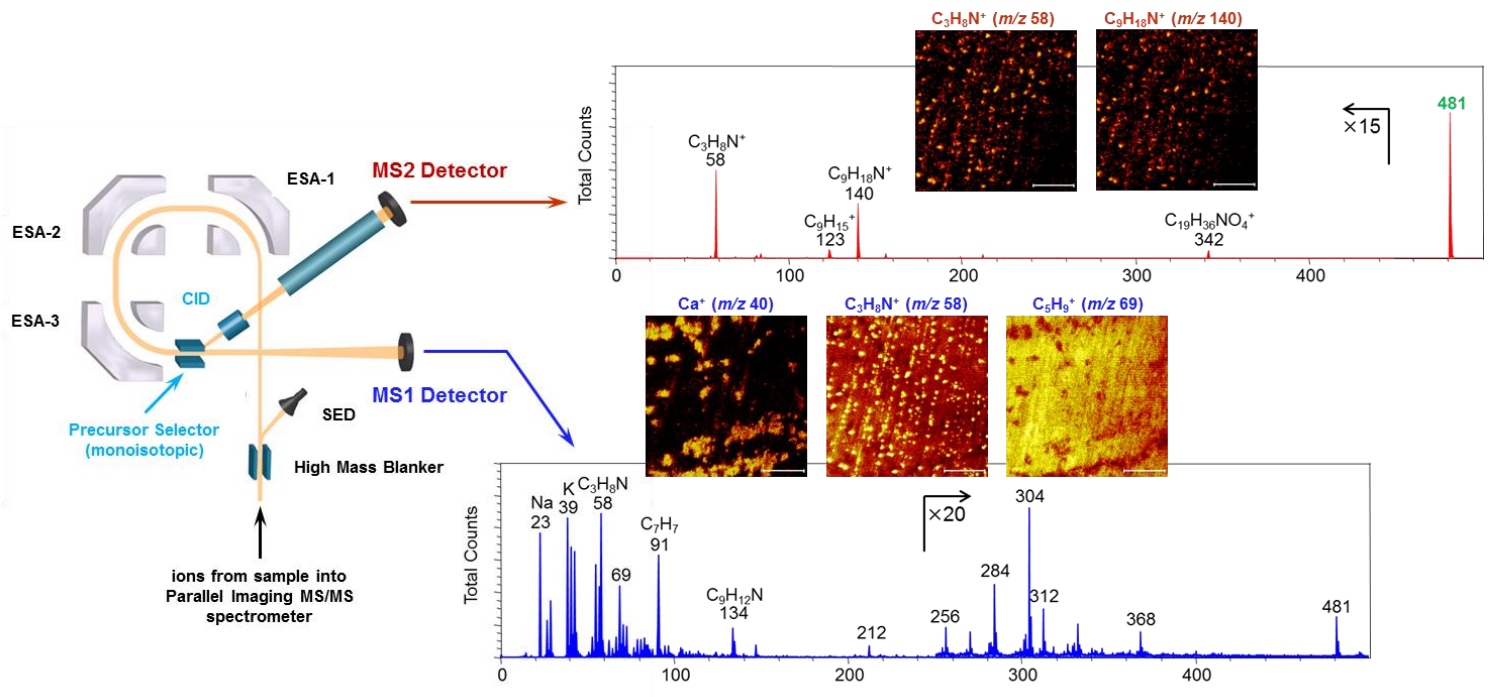

Figure 1. (LEFT) A schematic illustration of the TOF-TOF Parallel Imaging MS/MS spectrometer. The major components are identified. (BOTTOM-RIGHT) TOF-SIMS (MS ${ }^{1}$ ) precursor ion spectrum of a poly(propylene) specimen from an automotive component. Several images (inset) are generated from the corresponding $\mathrm{MS}^{1}$ precursor ion peaks. (TOP-RIGHT) Tandem MS (MS ${ }^{2}$ ) product ion spectrum of the $\mathrm{m} / \mathrm{z} 481.40$ precursor. Two images (inset) are generated from the corresponding $\mathrm{MS}^{2}$ product ion peaks. The TOF-SIMS tandem MS imaging data were acquired simultaneously in an 8 minute acquisition. 


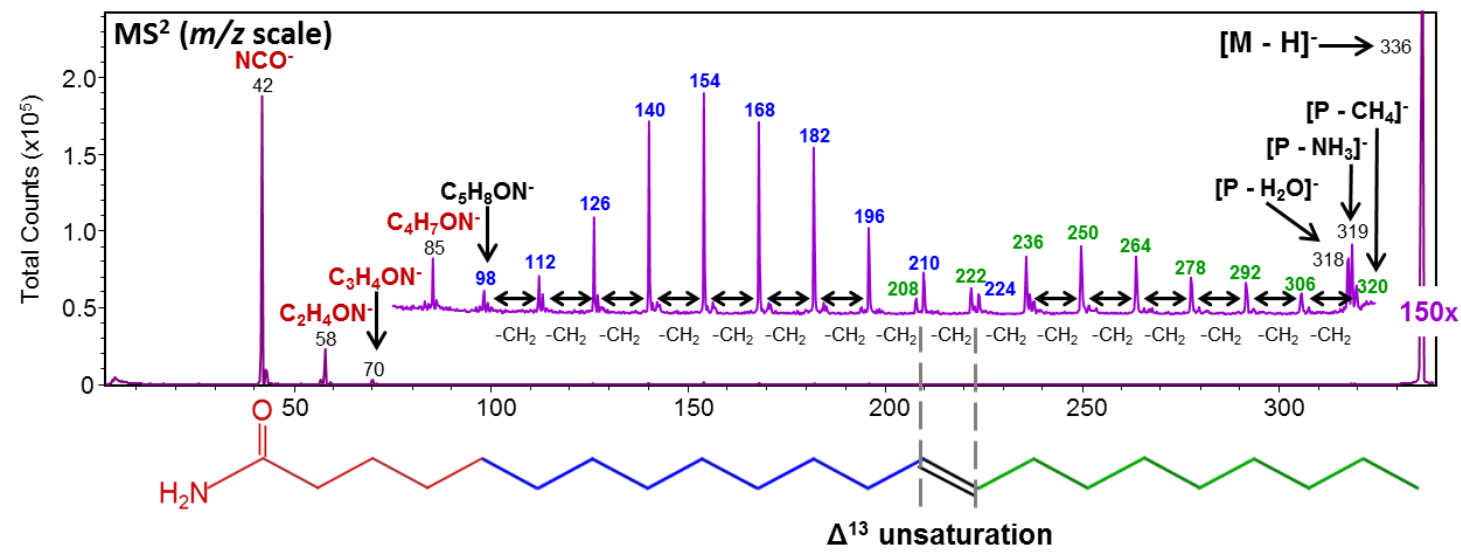

Figure 2. Tandem MS (MS $\left.{ }^{2}\right)$ product ion spectrum of the $[\mathrm{M}-\mathrm{H}]^{-}(\mathrm{m} / \mathrm{z} 336)$ precursor ions of erucamide. The $\mathrm{C}=\mathrm{C}$ double bond is clearly identified at the $\Delta^{13}$ position. A stick model of the erucamide molecule is shown below the $\mathrm{MS}^{2}$ spectrum, and the model has been scaled such that each molecular carbon atom is matched with the corresponding spectral feature. The detailed structural elucidation is a product of the remote-charge fragmentation mechanism that is enabled by the $\mathrm{keV}$ collision-induced dissociation. (Adapted from G.L. Fisher, A.L. Bruinen, N. Ogrinc Potočnik, J.S. Hammond, S.R. Bryan, P.E. Larson, R.M.A. Heeren, Anal. Chem. 88 (2016) 6433-6440.)
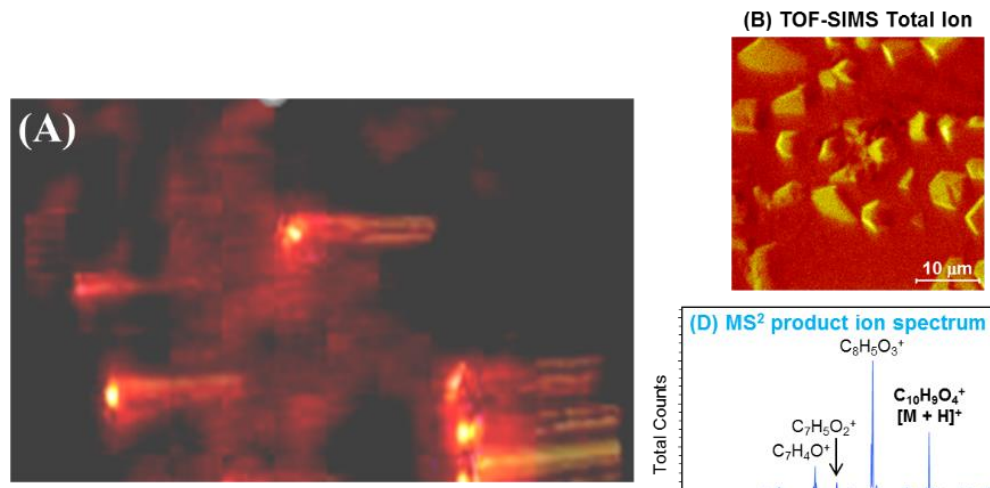

(C) Tandem MS Total Ion ( $\mathrm{m} / \mathbf{z}$ 577)
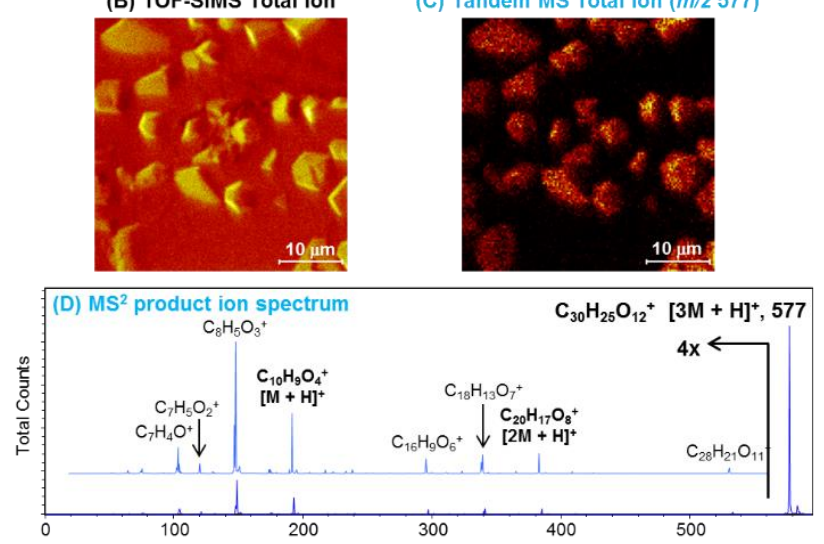

Figure 3. (A) Three-dimensional TOF-SIMS tandem MS image of endoplasmic reticulum (ER) tubules in a transfected human embryonic kidney (HEK) cell. Some ER tubules extend from the ER sheets to the plasma membrane (PM) forming ER-PM junctions. (B) TOF-SIMS (MS ${ }^{1}$ ) total ion image of heat-treated poly(ethylene terephthalate). (C) Tandem MS $\left(\mathrm{MS}^{2}\right)$ total ion image arising from the lateral distribution of the $m / z 577.14$ precursor. (D) Tandem MS $\left(\mathrm{MS}^{2}\right)$ product ion spectrum revealing the $m / z, 577.14$ precursor to be ethylene terephthalate trimer. The data in panels B - D were acquired simultaneously in a 13 minute acquisition (adapted from G.L. Fisher, J.S. Hammond, S.R. Bryan, P.E. Larson, R.M.A. Heeren, Microscop. Microanal. 23 (2017) 843-848). 